\title{
IDOSOS EM TRATAMENTO HEMODIALÍTICO: CARACTERÍSTICAS SOCIODEMOGRÁFICAS E SINTOMAS DEPRESSIVOS
}

Anderson Abreu de Carvalho; Departamento de Enfermagem da Universidade Federal de Santa Catarina

(UFSC); anderson.imbituba12@gmail.com

Bianca Dacoregio Martins; Departamento de Enfermagem da Universidade Federal de Santa Catarina

(UFSC); biancaamd@outlook.com

Naísa Falcão Martins; Departamento de Enfermagem da Universidade Federal de Santa Catarina (UFSC); naisa.falcom@live.com

Giordanna Nayara Chagas e Silva; Departamento de Enfermagem da Universidade Federal do Paraná (UFPR); giordanna@ufpr.br

Suzana Rosa; Departamento de Enfermagem da Universidade Federal de Santa Catarina (UFSC); suzana.srosa@hotmail.com

Juliana Balbinot Reis Girondi; Departamento de Enfermagem da Universidade Federal de Santa Catarina; juliana.balbinot@ufsc.br

Fernanda Rosa de Oliveira Pires; Departamento de Enfermagem da Universidade Federal de Santa Catarina (UFSC); nandadode@hotmail.com

Karina Silveira de Almeida Hammerschmidt; Departamento de Enfermagem da Universidade Federal do Paraná (UFPR); ksalmeidah@ufpr.br

\section{RESUMO}

Indrodução: $\mathrm{O}$ tratamento hemodialítico gera inúmeros impactos na vida do idoso, devido as mudanças decorrentes da doença, que podem gerar estressores físicos e psíquicos, influenciando o cotidiano de vida do idoso, torando-o propenso ao isolamento social, a diminuição de atividades diárias, incitando sintomas depressivos. Objetivo: Identificar as características sociodemográficas e sintomas depressivos de idosos em tratamento hemodialítico. Métodos: Quantitativo descritivo transversal, realizado com idosos em tratamento hemodialítico no sul do Brasil em 2018, através de entrevista gravada, utilizando instrumento contendo: dados socioeconômicos, de saúde e sintomas depressivos (Escala GDS). Para análise foi utilizada a ferramenta estatística BIOESTAT5.3. Aprovado no Comitê de Ética em Pesquisa, com número: 1097377. Resultados: Participaram da pesquisa 13 idosos, nove mulheres e quatro homens, com idade entre 60 a 78 anos, a maioria casadas, brancas, aposentadas, com renda de até dois salários e oito anos de estudo. Em relação a avaliação de depressão, verificou-se que 53,8\% apresentaram mais de cinco pontos na escala, evidenciando a percepção da presença de sintomas depressivos. Três apontam que os sintomas depressivos estão presentes na maior parte do dia e oito perceberam esses sintomas em outros momentos da vida. Concernente ao que o idoso realiza para amenizar os sintomas, quatro afirmam utilizar medicamentos antidepressivos, dois realizam terapia psicológica e nenhum menciona o 
uso de terapias alternativas. Conclusão: Verificou-se nesses idosos, indícios de sintomas depressivos. Este achado é importante para o cuidado de enfermagem, planejamento em saúde, assim como monitoramento de comorbidades, possibilitando melhor compreensão da qualidade de vida destes idosos.

Palavras-chave: Idoso; Insuficiência renal crônica; Perfil de saúde; Depressão. 